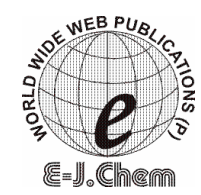

http://www.e-journals.net

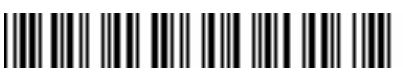

ISSN: 0973-4945; CODEN ECJHAO

E-Journal of Chemistry

Vol. 5, No. 1, pp. 114-119, January 2008

\title{
Synthesis and Characterization of New Diketone Analogues of Podophyllotoxin
}

\author{
K. H. HEMAKUMAR*, A. D. SATHISHA and Y. B. BASAVARAJU \\ Department of Studies in Chemistry, \\ University of Mysore, Manasagangotri, Mysore-570 006, Karnataka, India. \\ basavaraju_yb@yahoo.co.in
}

Received 2 June 2007; Accepted 13 July 2007

\begin{abstract}
The new compounds 6,6a-dihydro-2,3-dimethoxy-9-nitro- $11 \mathrm{bH}$ benzo [C]-fluoren 5,7-dione, 6,6a-dihydro-2,3-dimethoxy-9-chloro-11bH benzo[C]-fluoren-5,7-dione and 6,6a-dihydro-2,3-dimethoxy-9-fluoro-11bH benzo[C]-fluoren-5,7- dione were synthesized in high yields. They are analogues of naturally occurring lignan podophyllotoxin which exhibits anticancer activity. They are very essential to study anticancer activity.
\end{abstract}

Keywords: Benzophenones, Stobbe condensation, Itaconic acids, Sodium-amalgam, Benzhydryl succinic acids, Diketones.

\section{Introduction}

Podophyllotoxin $\mathbf{1}$ is a naturally occurring lignan compound, which has been isolated from the plants of genus Podophyllum ${ }^{1-4}$ belongs to the family of Berberidaceae. As the compound 1 was found to be highly cytotoxic for its clinical use against human cancers ${ }^{5}$, extensive structural modifications of $\mathbf{1}$ have been undertaken which culminated in to two semi-synthetic analogues of podophyllotoxin, namely etoposide (VP-16) and tenoposide (VM-26) are now in clinical use. Several analogues of podophyllotoxin have been synthesized with a view to study their structure activity relationship ${ }^{6}$. Hence, it was decided to synthesize analogues $2,3 \& 4$ by modifying the structure of podophyllotoxin 1 . Several synthetic routes ${ }^{7}$ have been reported for the synthesis of analogues of podophyllotoxin $\mathbf{1}$. In this context, we have chosen Gensler's ${ }^{8,9}$ method with some changes in the experimental procedure and reagents to synthesize diketone analogues.

\section{Experimental}

Melting points of the products were determined by the open capillary method and are uncorrected. The IR spectra were recorded on a FT-IR in KBr disc or in nujol mull. The ${ }^{1} \mathrm{H}$ NMR spectra were recorded on Jeol-60MHz and Jeol GSX $400 \mathrm{MHz}$ spectrophotometer 
using $\mathrm{CDCl}_{3}$ as a solvent and TMS as an internal reference. The chemical shifts are expressed in $\delta(\mathrm{ppm})$ values. The Mass spectra were recorded on Hitachi RMU-61 spectrophotometer and important fragments are given with percentage of abundance in the bracket. The purity of the compounds were checked by thin layer chromatography on silica gel glass plates in benzene and ethyl acetate solvent mixture (7:0.5 v/v). The compounds were purified by column chromatography using silica gel (60-120 mesh) as adsorbent and benzene as eluent.

\section{General procedure for the preparation of 3,4-dimethoxy-4'-nitro-benzophenone (7a)}

Veratrole (5) (10g, 0.0724mole) and anhydrous aluminum chloride $(9.650 \mathrm{~g}, 0.0724 \mathrm{~mole})$ were taken in dichloromethane $(75 \mathrm{~mL})$. The reaction mixture was cooled to $\mathrm{O}{ }^{0} \mathrm{C}$ and protected from atmospheric moisture. It was stirred continuously for $30 \mathrm{~min}$. A solution of $p$-nitro benzoyl chloride $(13.43 \mathrm{~g}, 0.0724 \mathrm{~mole})$ in dichloromethane $(75 \mathrm{~mL})$ was added drop wise over a period of $1 \mathrm{~h}$ to the above reaction mixture. After $12 \mathrm{~h}$, the temperature of the reaction mixture had been allowed to come to $25{ }^{\circ} \mathrm{C}$, conc. $\mathrm{HCl}(54 \mathrm{~mL})$ was added drop wise over a period of $30 \mathrm{~min}$. The reaction mixture was further stirred for $10 \mathrm{~h}$. During the addition of $\mathrm{HCl}$ and for some time thereafter, large amount of $\mathrm{HCl}$ gas is evolved. The product was extracted into chloroform, washed with $10 \%$ aqueous $\mathrm{NaOH}$ solution $(2 \times 100 \mathrm{~mL})$ and then with $2 \%$ aqueous $\mathrm{NaCl}$ solution $(2 \times 75 \mathrm{~mL})$. The solvent was removed by distillation. The product was recrystallized from methanol to give brown crystalline compound in $72.96 \%$ yield $(15.3 \mathrm{~g})$. M.p.143-145 ${ }^{0} \mathrm{C}$. IR (KBr): $1680(\mathrm{C}=\mathrm{O}), 1596$ (aromatic $\left.\mathrm{C}=\mathrm{C}\right) \mathrm{cm}^{-1} ;{ }^{1} \mathrm{H} \mathrm{NMR}\left(\mathrm{CDCl}_{3}\right): \delta 3.9(\mathrm{~s}$, $\left.6 \mathrm{H}, \mathrm{OCH}_{3}\right), 6.8\left(\mathrm{~m}, 3 \mathrm{H}, \mathrm{C}_{5}, \mathrm{C}_{3^{\prime}} \& \mathrm{C}_{5^{\prime}}-\mathrm{H}\right), 7.3\left(\mathrm{~m}, 4 \mathrm{H}, \mathrm{C}_{2}, \mathrm{C}_{6}, \mathrm{C}_{2^{\prime}} \& \mathrm{C}_{6^{\prime}}-\mathrm{H}\right)$; Anal Calcd. for $\mathrm{C}_{15} \mathrm{H}_{13} \mathrm{O}_{5} \mathrm{~N}$ : C, 62.72; H, 4.56; N, 5.22\%; Found: C, 62.70; H, 4.50; N, 5.19\%.

\section{3,4-Dimethoxy-4'-chloro-benzophenone (7b)}

Prepared from veratrole (5) $(10 \mathrm{~g}, 0.0724 \mathrm{~mole})$ and $p$-chloro benzoyl chloride $(10.10 \mathrm{~g}$, $0.0724 \mathrm{~mole})$ as yellow semisolid compound in $74 \%$ yield (14.93g). IR (Nujol): $1684(\mathrm{C}=\mathrm{O})$, 1594 (aromatic $\mathrm{C}=\mathrm{C}) \mathrm{cm}^{-1} ;{ }^{1} \mathrm{H} \mathrm{NMR}\left(\mathrm{CDCl}_{3}\right): \delta 3.9\left(\mathrm{~s}, 6 \mathrm{H}, \mathrm{OCH}_{3}\right), 6.7\left(\mathrm{~m}, 3 \mathrm{H}, \mathrm{C}_{5}, \mathrm{C}_{3^{\prime}} \& \mathrm{C}_{5^{\prime}}\right.$ $\mathrm{H}), 7.2\left(\mathrm{~m}, 4 \mathrm{H}, \mathrm{C}_{2}, \mathrm{C}_{6}, \mathrm{C}_{2}, \& \mathrm{C}_{6^{\prime}}-\mathrm{H}\right)$; Anal. Calcd. for $\mathrm{C}_{15} \mathrm{H}_{13} \mathrm{O}_{3} \mathrm{Cl} ; \mathrm{C}, 65.10 ; \mathrm{H}, 4.73 \%$; Found: C, 65.05; H, $4.70 \%$.

\section{3,4-Dimethoxy-4'-fluoro-benzophenone (7c)}

Prepared from veratrole (5) $(10 \mathrm{~g}, 0.0724 \mathrm{~mole})$ and $p$-fluoro benzoyl chloride $(8.91 \mathrm{~g}$, $0.0724 \mathrm{~mole})$ as buff coloured solid in $76 \%$ yield $(14.31 \mathrm{~g})$. M.p. $102-104^{\circ} \mathrm{C}$. IR (KBr): 1678 $(\mathrm{C}=0), 1599$ (aromatic $\mathrm{C}=\mathrm{C}) \mathrm{cm}^{-1} ;{ }^{1} \mathrm{H} \mathrm{NMR}\left(\mathrm{CDCl}_{3}\right): \delta 3.8\left(\mathrm{~s}, 6 \mathrm{H}, \mathrm{OCH}_{3}\right), 6.9\left(\mathrm{~m}, 3 \mathrm{H}, \mathrm{C}_{5}\right.$, $\left.\mathrm{C}_{3^{\prime}} \& \mathrm{C}_{5^{\prime}}-\mathrm{H}\right), 7.3\left(\mathrm{~m}, 4 \mathrm{H}, \mathrm{C}_{2}, \mathrm{C}_{6}, \mathrm{C}_{2^{\prime}} \& \mathrm{C}_{6^{\prime}} \mathrm{H}\right)$; Anal. Calcd. for $\mathrm{C}_{15} \mathrm{H}_{13} \mathrm{O}_{3} \mathrm{~F}: \mathrm{C}, 69.22 ; \mathrm{H}$, $5.03 \%$; Found: C, 69.20; H, 5.00\%.

\section{General procedure for the preparation of 3,4-dimethoxy-4'-nitro-diphenyl itaconic acid ( 9a)}

It is prepared by the Stobbe condensation of 3,4-dimethoxy-4'-nitro benzophenone (7a) $(15.0 \mathrm{~g}$, $0.0522 \mathrm{~mole})$ with diethyl succinate $(9.09 \mathrm{~g}, 0.0522 \mathrm{~mole})$ in presence of potassium $t$-butoxide (obtained from potassium $2.04 \mathrm{~g}, 0.0522$ mole and $t$-butyl alcohol) in $t$-butyl alcohol $(100 \mathrm{~mL})$ at reflux temperature for $10 \mathrm{~h}$. The cooled reaction mixture was treated with $5 \mathrm{~N}$ conc. $\mathrm{HCl}$ $(50 \mathrm{~mL})$ was concentrated to $60 \mathrm{~mL}$ and diluted with water $(75 \mathrm{~mL})$. The itaconic acid half esters were extracted into ether $(3 \times 50 \mathrm{~mL})$ and then into saturated sodium bicarbonate solution $(3 \times 50 \mathrm{~mL})$. The bicarbonate solution was acidified with conc. $\mathrm{HCl}$ to give a brown crystalline solid in $83.9 \%$ yield $(18.2 \mathrm{~g})$. It was recrystallised from ethanol to give a pale brown crystalline solid. M.p. $97-98{ }^{\circ} \mathrm{C}$. The itaconic acid half esters were saponified by refluxing in methanol $(50 \mathrm{~mL})$ and water $(50 \mathrm{~mL})$ mixture containing $\mathrm{NaOH}(6 \mathrm{~g})$. The reaction 
mixture was acidified with conc. $\mathrm{HCl}$ to give a grayish white solid. It was recrystallized from methanol to give white solid in $86 \%$ yield $(14.43 \mathrm{~g})$. M.p. $91-93{ }^{\circ} \mathrm{C}$. IR $(\mathrm{KBr}): 3600$ 3300 (Carboxylic $\mathrm{OH}), 1700\left(-\mathrm{CH}_{2}-\mathrm{C}=\mathrm{O}\right), 1680(\alpha, \beta$ - unsaturated $\mathrm{C}=\mathrm{O}), 1590$ (aromatic $\mathrm{C}=\mathrm{C}), 1610-1605$ (conjugated $\mathrm{C}=\mathrm{C}) \mathrm{cm}^{-1} ;{ }^{1} \mathrm{H} \mathrm{NMR}\left(\mathrm{CDCl}_{3}\right): \delta 3.9\left(\mathrm{~s}, 6 \mathrm{H}, \mathrm{OCH}_{3}\right), 3.6(\mathrm{~s}$, $\left.2 \mathrm{H}, \mathrm{CH}_{2}\right), 6.7\left(\mathrm{~m}, 3 \mathrm{H}, \mathrm{C}_{3^{\prime}}, \mathrm{C}_{5^{\prime}} \& \mathrm{C}_{5}-\mathrm{H}\right), 7.1\left(\mathrm{~m}, 4 \mathrm{H}, \mathrm{C}_{2}, \mathrm{C}_{6}, \mathrm{C}_{2} \& \mathrm{C}_{6}-\mathrm{H}\right)$; Anal. cald. for $\mathrm{C}_{19} \mathrm{H}_{17} \mathrm{O}_{8} \mathrm{~N}$ : C, 58.91; H, 4.42; N, $3.87 \%$; Found: C, 58.89; H, 4.40; N, 3.83\%.

\section{3,4-Dimethoxy-4'-chloro-diphenyl itaconic acid (9b)}

Prepared as white crystalline solid in $82 \%$ yield $(12.97 \mathrm{~g})$. M.p. $158{ }^{0} \mathrm{C}$, by the Stobbe condensation of 3,4-methoxy-4'-chloro benzophenone (7b) $(14 \mathrm{~g}, 0.0506 \mathrm{~mole})$ with diethyl succinate $(8.81 \mathrm{~g}, 0.0505 \mathrm{~mole})$ followed by saponification. IR (KBr): 3560-3330 (carboxylic $\mathrm{OH}), 1698\left(-\mathrm{CH}_{2}-\mathrm{C}=\mathrm{O}\right), 1672(\alpha, \beta$-unsaturated $\mathrm{C}=\mathrm{O}), 1598$ (conjugated $\left.\mathrm{C}=\mathrm{C}\right), 1593$ (aromatic $\mathrm{C}=\mathrm{C}) \mathrm{cm}^{-\mathrm{I}} ;{ }^{1} \mathrm{H}$ NMR $\left(\mathrm{CDCl}_{3}\right): \delta 3.8\left(\mathrm{~s}, 6 \mathrm{H}, \mathrm{OCH}_{3}\right), 3.7\left(\mathrm{~s}, 2 \mathrm{H}, \mathrm{CH}_{2}\right), 6.8(\mathrm{~m}, 3 \mathrm{H}$, $\left.\mathrm{C}_{3^{\prime}} \mathrm{C}_{5^{\prime}}, \& \mathrm{C}_{5^{-}}-\mathrm{H}\right), 7.2\left(\mathrm{~m}, 4 \mathrm{H}, \mathrm{C}_{2^{\prime}}, \mathrm{C}_{6^{\prime}}, \mathrm{C}_{2^{\prime}} \& \mathrm{C}_{6}-\mathrm{H}\right)$; Anal.cald. for $\mathrm{C}_{19} \mathrm{H}_{17} \mathrm{O}_{6} \mathrm{Cl}: \mathrm{C}, 60.56 ; \mathrm{H}$, 4.54\%; Found: C, 60.53; H, $4.50 \%$.

\section{3,4-Dimethoxy-4'-fluoro-diphenyl itaconic acid (9c)}

Prepared as white solid in $81.8 \%$ yield $\left(12.9\right.$ g). M.p. $136{ }^{\circ} \mathrm{C}$ by the Stobbe condensation of 3,4-methoxy-4'-fluoro benzophenone $(7 \mathrm{c})(14 \mathrm{~g}, 0.0538 \mathrm{~mole})$ with diethyl succinate $(9.37 \mathrm{~g}$, 0.0538 mole) followed by saponification. IR $(\mathrm{KBr}): 3500-3260$ (carboxylic $\mathrm{OH}), 1592$ (conjugated $\mathrm{C}=\mathrm{C}), 1603$ (aromatic $\mathrm{C}=\mathrm{C}), 1693\left(-\mathrm{CH}_{2}-\mathrm{C}=\mathrm{O}\right), 1680(\alpha, \beta$-unsaturated $\mathrm{C}=\mathrm{O}) \mathrm{cm}^{-1} ;{ }^{1} \mathrm{H}$ NMR $\left(\mathrm{CDCl}_{3}\right): \delta 3.9\left(\mathrm{~s}, 6 \mathrm{H}, \mathrm{OCH}_{3}\right), 3.6\left(\mathrm{~s}, 2 \mathrm{H}, \mathrm{CH}_{2}-\right), 6.7\left(\mathrm{~m}, 3 \mathrm{H}, \mathrm{C}_{3^{\prime}}, \mathrm{C}_{5^{\prime}} \& \mathrm{C}_{5}-\mathrm{H}\right), 7.3\left(\mathrm{~m}, 4 \mathrm{H}, \mathrm{C}_{2}, \mathrm{C}_{6}, \mathrm{C}_{2^{\prime}}\right.$ \& $\left.\mathrm{C}_{6}{ }^{\prime} \mathrm{H}\right)$; Anal. cald. for $\mathrm{C}_{19} \mathrm{H}_{17} \mathrm{O}_{5} \mathrm{~F}$ : C, 63.32; H, 4.76\%; Found: C, 63.29; H, 4.74\%.

General procedure for the preparation of 3,4-dimethoxy-4'-nitro benzhydryl succinic acid (10a)

Powdered 5\% sodium-amalgam (200g) was added to a solution of $9 \mathbf{a}(14.07 \mathrm{~g}, 0.0361 \mathrm{~mole})$ in $5 \%$ aq. $\mathrm{NaOH}(200 \mathrm{~mL})$ solution around $5{ }^{\circ} \mathrm{C}$. The reaction mixture was kept overnight at room temperature and filtered. The filtrate was acidified with $5 \mathrm{~N} \mathrm{HCl}$ gave gray solid. It was recrystallized from ethanol gave white solid in $81 \%$ yield $(11.39 \mathrm{~g})$. M.p. $123-125^{\circ} \mathrm{C}$. IR (KBr) :3500-3200 (carboxylic OH), 1710 (carboxyl $\mathrm{C}=\mathrm{O}$ ), 1590 (aromatic $\mathrm{C}=\mathrm{C}$ ) $\mathrm{cm}^{-1} ;{ }^{1} \mathrm{H}$ NMR $\left(\mathrm{CDCl}_{3}\right): \delta 3.9\left(\mathrm{~s}, 6 \mathrm{H}, \mathrm{OCH}_{3}\right), 3.0\left(\mathrm{~d}, \mathrm{~J}=6 \mathrm{~Hz}, 2 \mathrm{H}, \mathrm{C}_{\mathrm{c}}-\mathrm{H}\right), 3.5\left(\mathrm{q}, \mathrm{J}=6 \mathrm{~Hz}, 1 \mathrm{H}, \mathrm{C}_{\mathrm{b}}-\mathrm{H}\right), 4.1$ $\left(\mathrm{d}, \mathrm{J}=6 \mathrm{~Hz}, \mathrm{IH}, \mathrm{C}_{\mathrm{a}}-\mathrm{H}\right), 6.7\left(\mathrm{~m}, 3 \mathrm{H}, \mathrm{C}_{3}, \mathrm{C}_{5^{\prime}} \& \mathrm{C}_{5}-\mathrm{H}\right), 7.3\left(\mathrm{~m}, 4 \mathrm{H}, \mathrm{C}_{2}, \mathrm{C}_{6}, \mathrm{C}_{2^{\prime}} \& \mathrm{C}_{6^{\prime}}-\mathrm{H}\right)$; Anal.calcd. for $\mathrm{C}_{19} \mathrm{H}_{19} \mathrm{O}_{8} \mathrm{~N}$ : C, 58.61; H, 4.92; N, 3.85\%; Found: C, 58.59; H, 4.90; N, 3.80\%.

\section{3,4-Dimethoxy-4'-chloro benzhydryl succinic acid (10b)}

Obtained from the reduction of 3,4-dimethoxy-4'-chloro diphenyl itaconic acid 9b (12g, $0.0318)$ and $5 \%$ sodium-amalgam $(200 \mathrm{~g})$ as white solid in $79 \%$ yield $(9.53 \mathrm{~g})$. M.p. 117-119 ${ }^{0} \mathrm{C}$. IR (KBr) :3500-3250 (carboxylic OH), 1705 (carboxyl C=O), 1598 (aromatic $\mathrm{C}=\mathrm{C}$ ) $\mathrm{cm}^{-1} ;{ }^{1} \mathrm{H} \mathrm{NMR}\left(\mathrm{CDCl}_{3}\right): \delta 3.9\left(\mathrm{~s}, 6 \mathrm{H}, \mathrm{OCH}_{3}\right), 2.9\left(\mathrm{~d}, \mathrm{~J}=5 \mathrm{~Hz}, 2 \mathrm{H}, \mathrm{C}_{\mathrm{c}}-\mathrm{H}\right), 3.3(\mathrm{q}, \mathrm{J}=5 \mathrm{~Hz}, 1 \mathrm{H}$, $\left.\mathrm{C}_{\mathrm{b}}-\mathrm{H}\right), 4.0\left(\mathrm{~d}, \mathrm{~J}=5 \mathrm{~Hz}, 1 \mathrm{H}, \mathrm{C}_{\mathrm{a}}-\mathrm{H}\right), 7.2\left(\mathrm{~m}, 4 \mathrm{H}, \mathrm{C}_{2}, \mathrm{C}_{6}, \mathrm{C}_{2^{\prime}} \& \mathrm{C}_{6^{\prime}}-\mathrm{H}\right), 6.6\left(\mathrm{~m}, 3 \mathrm{H}, \mathrm{C}_{3^{\prime}}, \mathrm{C}_{5^{\prime}}\right.$ \& $\mathrm{C}_{5}-\mathrm{H}$ ); Anal.calcd. for $\mathrm{C}_{19} \mathrm{H}_{19} \mathrm{O}_{6} \mathrm{Cl}$ : C, 60.24; H, 5.05\%; Found: C, 60.19; H, 5.02\%.

\section{3,4-Dimethoxy-4'-fluoro benzhydryl succinic acid (10c)}

Obtained from the reduction of 3,4-dimethoxy-4'-fluoro diphenyl itaconic acid 9c $(12 \mathrm{~g}$, 0.0333 mole) and $5 \%$ sodium-amalgam $(200 \mathrm{~g})$ as white amorphous solid in $82.4 \%$ yield $(9.94 \mathrm{~g})$. M.p. $110-112{ }^{\circ} \mathrm{C}$. IR (KBr) :3600-3200 (carboxylic OH), 1700 (carboxyl C=O), 1610 (aromatic $\mathrm{C}=\mathrm{C}) \mathrm{cm}^{-1} ;{ }^{1} \mathrm{H} \mathrm{NMR}\left(\mathrm{CDCl}_{3}\right): \delta 3.9\left(\mathrm{~s}, 6 \mathrm{H}, \mathrm{OCH}_{3}\right), 2.8\left(\mathrm{~d}, \mathrm{~J}=6 \mathrm{~Hz}, 2 \mathrm{H}, \mathrm{C}_{\mathrm{c}}-\mathrm{H}\right), 3.4(\mathrm{q}, \mathrm{J}=6 \mathrm{~Hz}$, 
$\left.1 \mathrm{H}, \mathrm{C}_{\mathrm{b}}-\mathrm{H}\right), 4.1\left(\mathrm{~d}, \mathrm{~J}=6 \mathrm{~Hz}, 1 \mathrm{H}, \mathrm{C}_{\mathrm{a}}-\mathrm{H}\right), 7.3\left(\mathrm{~m}, 4 \mathrm{H}, \mathrm{C}_{2}, \mathrm{C}_{6}, \mathrm{C}_{2^{\prime}} \& \mathrm{C}_{6^{-}}-\mathrm{H}\right), 6.7\left(\mathrm{~m}, 3 \mathrm{H}, \mathrm{C}_{3^{\prime}}, \mathrm{C}_{5^{\prime}}\right.$ \& $\mathrm{C}_{5}-\mathrm{H}$ ); Anal.calcd. for $\mathrm{C}_{19} \mathrm{H}_{19} \mathrm{O}_{6} \mathrm{~F}: \mathrm{C}, 62.97 ; \mathrm{H}, 4.73 \%$; Found: C, 62.94; H, 4.69\%

General procedure for the preparation of 6,6a-dihydro-2,3-dimethoxy-9-nitro11 bH benzo [C]-fluoren-5,7-dione (2)

A mixture of 3,4-dimethoxy-4'-nitro benzhydryl succinic acid 10a $(2.0 \mathrm{~g}, 0.0051 \mathrm{~mole})$ and thionyl chloride $(40 \mathrm{~mL})$ was refluxed for $5 \mathrm{~h}$. The excess thionyl chloride was distilled off. A pale yellow solid was obtained as gummy product 11a in $80 \%$ yield $(1.74 \mathrm{~g})$. A solution of 3,4 dimethoxy-4'-nitro benzhydryl succinyl chloride 11a $(1.6 \mathrm{~g}, 0.0038 \mathrm{~mole})$ in dry dichloromethane $(50 \mathrm{~mL})$ was added over a period of $20 \mathrm{~min}$ to a stirred solution of anhydrous aluminium chloride $(0.50 \mathrm{~g}, 0.0037 \mathrm{~mole})$ in dry dichloromethane at $0{ }^{\circ} \mathrm{C}$. The reaction mixture was further stirred at $0{ }^{\circ} \mathrm{C}$ for $6 \mathrm{~h}$. After the reaction, the reaction mixture was treated with cold $5 \mathrm{~N} \mathrm{HCl}(50 \mathrm{~mL})$. The organic layer was washed with $10 \% \mathrm{NaOH}$ solution $(2 \times 50 \mathrm{~mL})$ and finally with water. The solvent was removed by distillation to get a brown solid. The crude product was column chromatographed over silica gel $(1 \mathrm{~cm} \times 30 \mathrm{~cm})$ using chloroform as the eluent. The solvent was removed at $50{ }^{\circ} \mathrm{C}$ on a rotary evaporator to get a pale brown solid. It was recrystallized from ethanol gave $72.3 \%$ yield $(0.96 \mathrm{~g})$. M.p. $163-164{ }^{\circ} \mathrm{C}$. IR (KBr): 1743 (Indonone carbonyl), 1704 (tetralone carbonyl), 1594 (aromatic $\mathrm{C}=\mathrm{C}$ ) $\mathrm{cm}^{-1} ;{ }^{1} \mathrm{H} \mathrm{NMR}\left(\mathrm{CDCl}_{3}\right)$ : $\delta 2.5\left(\mathrm{dd}, \mathrm{J}=4 \mathrm{~Hz}, 2 \mathrm{H}, \mathrm{C}_{6}-\mathrm{H}\right), 3.4\left(\mathrm{q}, \mathrm{J}=4 \mathrm{~Hz}, 1 \mathrm{H}, \mathrm{C}_{6 \mathrm{a}} \mathrm{-H}\right), 3.8\left(\mathrm{~s}, 6 \mathrm{H}, \mathrm{OCH}_{3}\right), 4.2(\mathrm{~d}, \mathrm{~J}=6 \mathrm{~Hz}, 1 \mathrm{H}$, $\left.\mathrm{C}_{11 \mathrm{~b}}-\mathrm{H}\right), 7.5\left(\mathrm{~s}, 1 \mathrm{H}, \mathrm{C}_{8}-\mathrm{H}\right), 7.3\left(\mathrm{~s}, 1 \mathrm{H}, \mathrm{C}_{4}-\mathrm{H}\right), 6.7\left(\mathrm{~m}, 3 \mathrm{H}, \mathrm{C}_{1}-\mathrm{H}, \mathrm{C}_{10}-\mathrm{H} \& \mathrm{C}_{11}-\mathrm{H}\right) ;$ Mass $(\mathrm{m} / \mathrm{z} \%$ abundance): 353 ( $\left.\mathrm{M}^{+}, 24\right), 325$ (46), 298 (27), 270 (33), 135 (69), 89 (93). Anal. Calcd. for $\mathrm{C}_{19} \mathrm{H}_{14} \mathrm{O}_{6} \mathrm{~N}$ : C, 63.74; H, 3.99; N, 4.24\%; Found: C, 63.69; H, 3.97: N, 4.20\%.

\section{6,6a Dihydro-2,3-dimethoxy-9-chloro 11 bH benzo [C]-fluoren-5,7-dione (3)}

Prepared from 3,4-dimethoxy-4'-fluoro benzhydryl succinic acid 10b $(2.0 \mathrm{~g}, 0.0538 \mathrm{~mole})$ thionyl chloride $(40 \mathrm{~mL})$ as brown semisolid $\mathbf{1 1 b}$ in $78.2 \%$ yield $(1.70 \mathrm{~g})$. The $\mathbf{1 1 b}(1.60 \mathrm{~g}$ 0.0038 mole) was cyclised to 3 using anhyd. aluminium chloride $(0.52 \mathrm{~g}, 0.0038 \mathrm{~mole})$ in dry dichloromethane $(50 \mathrm{~mL})$ to get pale yellow semi solid compound in $67 \%$ yield $(0.87 \mathrm{~g})$. IR $(\mathrm{KBr})$ : 1740(Indanone $\mathrm{C}=\mathrm{O}$ ), 1696 (tetralone $\mathrm{C}=\mathrm{O}$ ), 1598 (aromatic $\mathrm{C}=\mathrm{C}$ ) $\mathrm{cm}^{-1}$; ${ }^{1} \mathrm{H}$ NMR $\left(\mathrm{CDCl}_{3}\right): \delta 2.7\left(\mathrm{dd}, \mathrm{J}=4 \mathrm{~Hz}, 2 \mathrm{H}, \mathrm{C}_{6}-\mathrm{H}\right), 3.4\left(\mathrm{q}, \mathrm{J}=4 \mathrm{~Hz}, 1 \mathrm{H}, \mathrm{C}_{6 \mathrm{a}}-\mathrm{H}\right), 3.8\left(\mathrm{~s}, 6 \mathrm{H}, \mathrm{OCH}_{3}\right), 4.2(\mathrm{~d}$, $\left.\mathrm{J}=4 \mathrm{~Hz}, 1 \mathrm{H}, \mathrm{C}_{11 \mathrm{~b}}-\mathrm{H}\right), 7.4\left(\mathrm{~s}, 1 \mathrm{H}, \mathrm{C}_{8}-\mathrm{H}\right), 7.2\left(\mathrm{~s}, 1 \mathrm{H}, \mathrm{C}_{4}-\mathrm{H}\right), 6.7-7.1\left(\mathrm{~m}, 3 \mathrm{H}, \mathrm{C}_{1}-\mathrm{H}, \mathrm{C}_{10}-\mathrm{H} \&\right.$ $\left.\mathrm{C}_{11}-\mathrm{H}\right)$; Mass (m/z \% of abundance): $342.5\left(\mathrm{M}^{+}, 19\right), 307$ (26), 279 (44), 252 (37), 224 (32), 89 (76); Anal. Calcd. for $\mathrm{C}_{19} \mathrm{H}_{14} \mathrm{O}_{4} \mathrm{Cl}$ : C, 66.48; H, 4.16\%; Found: C, 66.44; H, 4.13\%.

\section{6,6a, Dihydro-2,3-dimethoxy-9-fluoro-11bH benzo [C]-fluoren-5,7-dione 4}

Prepared from 3,4-dimethoxy-4'-fluoro benzhydryl succicinic acid 10c $(2.0 \mathrm{~g}, 0.0055 \mathrm{~mole})$ and thionyl chloride $(40 \mathrm{~mL})$ as brown semi solid 11c in $74.1 \%$ yield $(1.62 \mathrm{~g})$. The $11 \mathrm{c}(1.6 \mathrm{~g}$, $0.0040 \mathrm{~mole})$ was cyclised to 4 using anhyd. aluminium chloride $(0.54 \mathrm{~g}, 0.0040 \mathrm{~mole})$ as catalyst in dry dichloromethane $(50 \mathrm{~mL})$ gave pale brown semi solid compound in $59.7 \%$ yield $(0.78 \mathrm{~g})$. IR (KBr): 1743 (Indanone $\mathrm{C}=\mathrm{O}$ ), 1693 (tetralone $\mathrm{C}=\mathrm{O}$ ), 1606 (aromatic $\mathrm{C}=\mathrm{C}$ ) $\mathrm{cm}^{-1} ;{ }^{1} \mathrm{H}$ NMR $\left(\mathrm{CDCl}_{3}\right): \delta 2.6\left(\mathrm{dd}, \mathrm{J}=4 \mathrm{~Hz}, 2 \mathrm{H}, \mathrm{C}_{6}-\mathrm{H}\right), 3.5\left(\mathrm{q}, \mathrm{J}=4 \mathrm{~Hz}, 1 \mathrm{H}, \mathrm{C}_{6 \mathrm{a}}-\mathrm{H}\right), 3.9\left(\mathrm{~s}, 6 \mathrm{H}, \mathrm{OCH}_{3}\right), 4.0(\mathrm{~d}, \mathrm{~J}=$ $\left.4 \mathrm{~Hz}, 1 \mathrm{H}, \mathrm{C}_{11 \mathrm{~b}}-\mathrm{H}\right), 7.7\left(\mathrm{~s}, 1 \mathrm{H}, \mathrm{C}_{8}-\mathrm{H}\right), 7.3\left(\mathrm{~s}, 1 \mathrm{H}, \mathrm{C}_{4}-\mathrm{H}\right), 6.8\left(\mathrm{~m}, 3 \mathrm{H}, \mathrm{C}_{1}-\mathrm{H}, \mathrm{C}_{10}-\mathrm{H} \& \mathrm{C}_{11}-\mathrm{H}\right) ;$ Mass (m/z \% of abundance): $326\left(\mathrm{M}^{+}, 23\right), 298$ (37), 271 (43), 243 (17), 108 (54), 89 (83); Anal. Calcd. for $\mathrm{C}_{19} \mathrm{H}_{14} \mathrm{O}_{4} \mathrm{~F}$; C, 69.22; H, 4.33\%; Found: C, 69.19; H, $4.29 \%$.

\section{Results and Discussion}

The starting materials benzophenones 7a-c Were prepared in high yields by Friedel-Crafts acylation of veratrole with acyl chlorides 6a-c in the presence of anhydrous aluminum 
chloride in dry dichloromethane at $0{ }^{0} \mathrm{C}$. Itaconic acid half esters 8a-c were prepared as a mixture of cis and trans isomers in good yields by Stobbe condensation of benzophenones 7a-c with diethyl succinate using potassium t-butoxide as a base in $t$-butanol at $90{ }^{\circ} \mathrm{C}$ (Scheme-1).

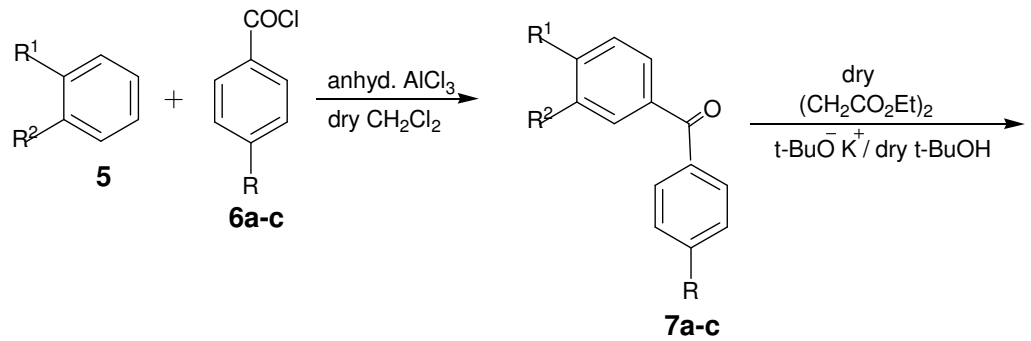

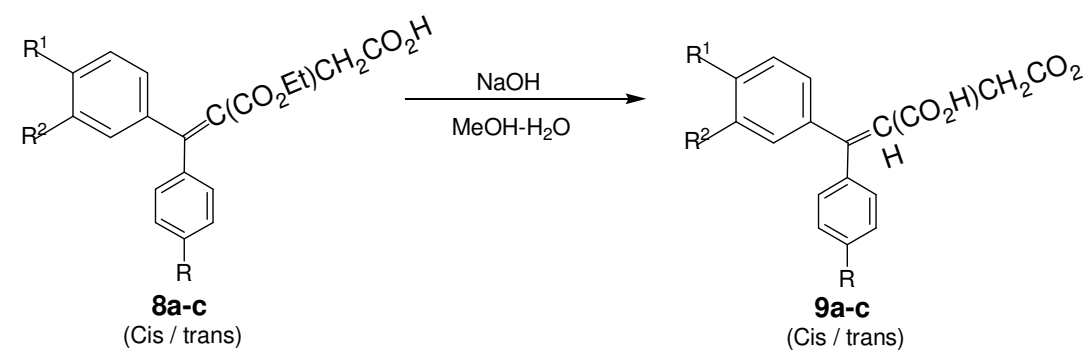

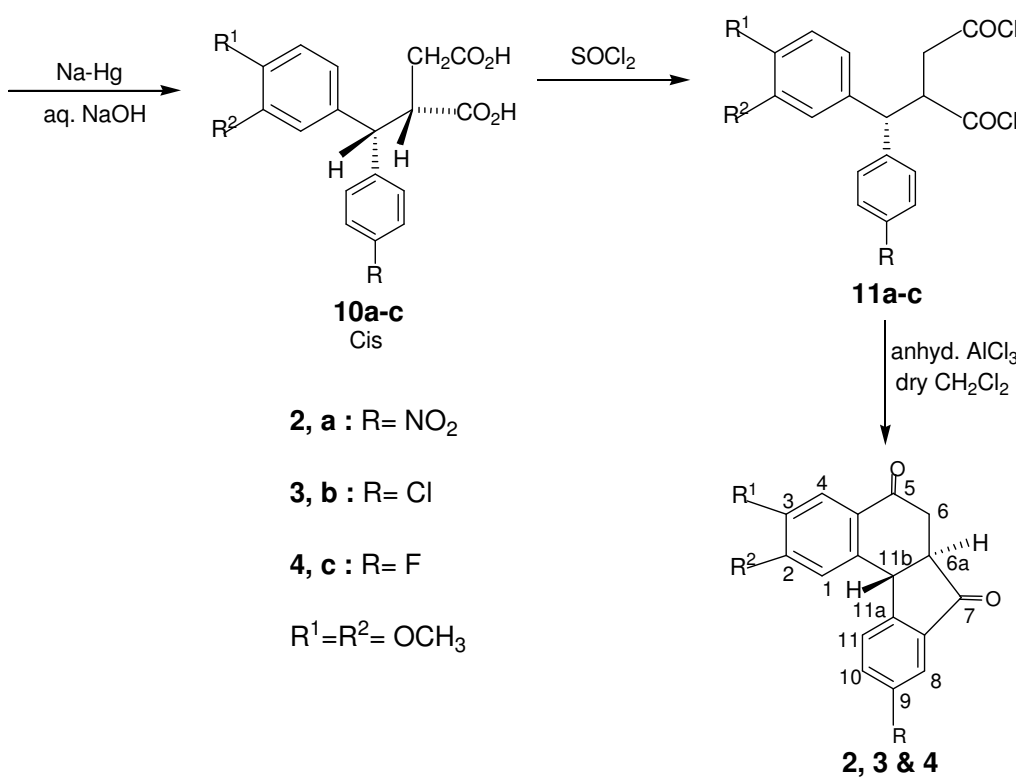

\section{Scheme 1}

Itaconic acids 9a-c were prepared in excellent yield by the saponification of itaconic acid half esters 8a-c. The separation of isomeric mixture by fractional crystallization from ethyl acetate-petroleum ether (60-80) mixture was unsuccessful. Hence, benzhydryl succinic acids 
10a-c were prepared by the reduction ${ }^{10}$ of isomeric mixture of itaconic acids 9a-c with $5 \%$ sodium-amalgam in $5 \%$ aq. sodium hydroxide solution. After the usual workup, only one required benzhydryl succinic acid isomer ${ }^{10}$ is formed in good yield. The compounds 11a-c were prepared by refluxing benzhydryl succinic acids $\mathbf{1 0 a - c}$ with thionyl chloride. Diketones $\mathbf{2}, 3 \& \mathbf{4}$ analogues of podophyllotoxin were prepared in good yields by the intramolecular cyclisation of benzhydryl succinyl chlorides 11a-c in the presence of anhydrous aluminium chloride in dichloromethane. The products were characterized by IR, ${ }^{1} \mathrm{H}$ NMR and mass spectral and elemental analysis data. The proton signals of carboxylic acids are not observed due to out of scale absorption (810.5-12.0). However, the acid functional groups were characterized by simple chemical tests. The NMR spectra of diketone $\mathbf{2}$ showed a doublet at $\delta 4.1 \mathrm{ppm}(\mathrm{J}=6 \mathrm{~Hz})$ for the benzylic proton $\mathrm{C}_{4}-\mathrm{H}$. The large coupling constant $(\mathrm{J})$ value indicated that $\mathrm{C}_{6 \mathrm{a}}-\mathrm{H}$ and $\mathrm{C}_{11 \mathrm{~b}}-\mathrm{H}$ were diaxial. Hence, the two ketone rings are trans fused and configuration being thermodynamically stable.

\section{Conclusion}

In the above synthetic scheme, the products are formed in good yields using less expensive and readily available chemicals. The double bond of the $\alpha, \beta$-unsaturated acids can be easily reduced to benzhydryl succinic acids. Benzhydryl succinyl chlorides undergo readily Friedel-Crafts intramolecular cyclisation in the presence of anhyd. aluminium chloride in dry dichloromethane gave high yields of diketone analogues of podophyllotoxin. They are required for studying anticancer activity.

\section{Acknowledgement}

Authors are grateful to the CDRI, Lucknow and IIT Madras, Chennai, India for providing spectral and elemental analysis data of our research compounds.

\section{References}

1. Podwyssotzki V, Arch. Exp. pathol. Parmakol., 1880, 13, 29.

2. Hartwell J L and Schrecker A W Fortschr Chem. Org. Naturist, 1958, 13, 83.

3. David Jackson E and Paul Dewick M, Phytochemistry, 1985, 24, 2407.

4. Hamburger M, Marston A and Hostettmann K, Adv. Drug Res., 1991, 20, 175.

5. (a) Alton P A and Harris A L, Annulation Br. J. Haematol.1993, 85, 241-245.

(b) Liu L F and Wang J C, Biochemistry of DNA Topoisomerases and their poisons.

In DNA Topoisomerases in Cancer, Potonesil $\mathrm{M}$ and Kohn $\mathrm{K} \mathrm{W}$ Ed. Oxford University Press: New York 1991, pp 13-22.

6. Kelly M G and Hartwell J L, J. Natl. Cancer Inst. 1961, 14, 459.

7. Ward R S, Chem. Soc. Rev. 1982, 11, 75; idem, synthesis, 1992, 719.

8. Basavaraju Y B and Anjanamurthy C, Ind. J. Chem. 2003, 42B, 876.

9. Gensler W J, Samour C M, Wang S Y and Johnson F, J. Am. Chem. Soc. 1960 82, 1714.

10. Shirvaiker A S and Kulkarni A B, Indian J. Chem. 1979, 17B, 198. 


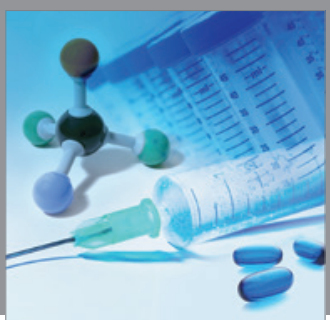

International Journal of

Medicinal Chemistry

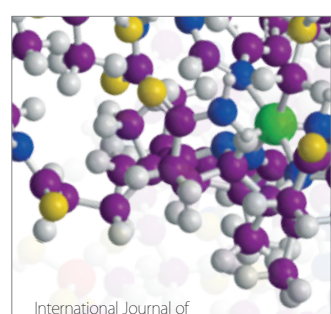

Carbohydrate Chemistry

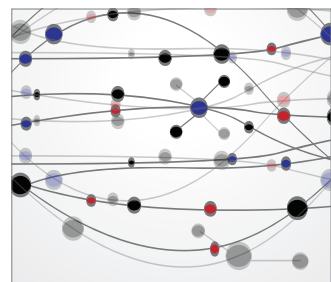

The Scientific World Journal
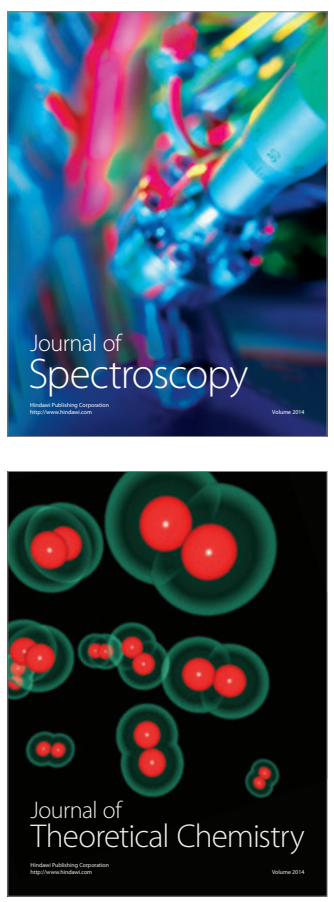
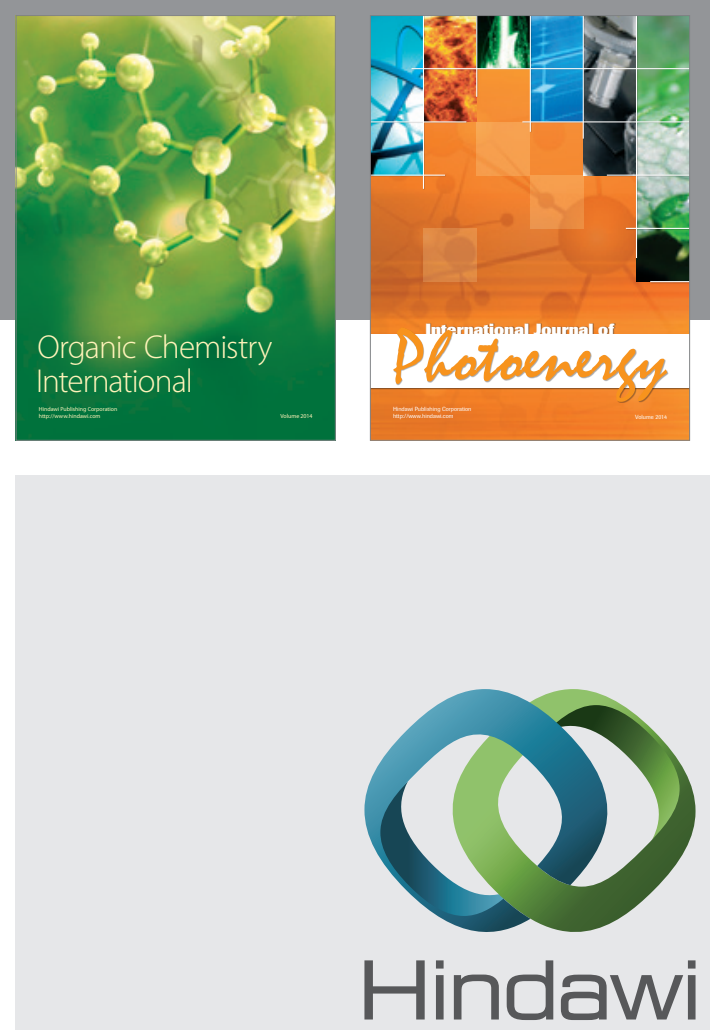

Submit your manuscripts at

http://www.hindawi.com
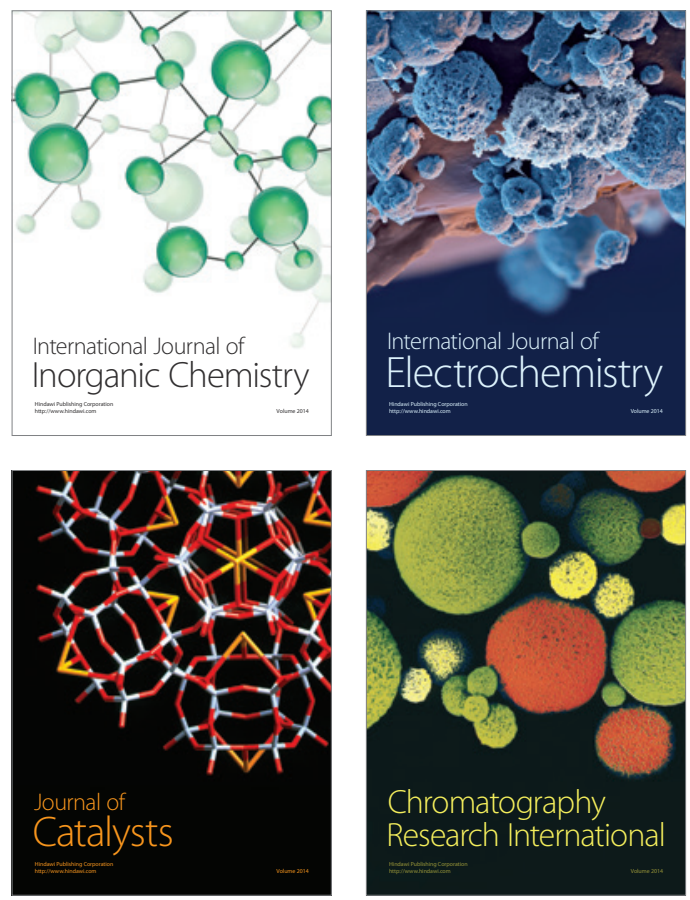
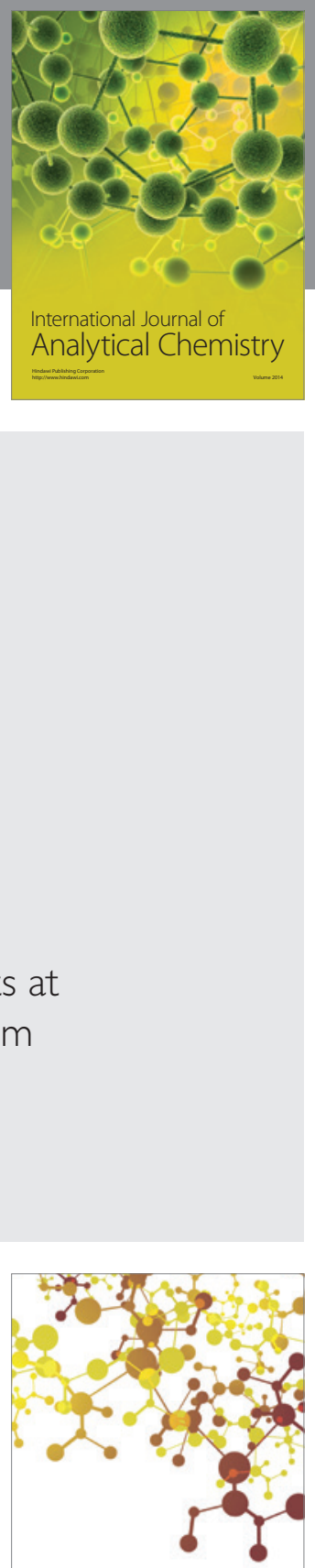

Journal of

Applied Chemistry
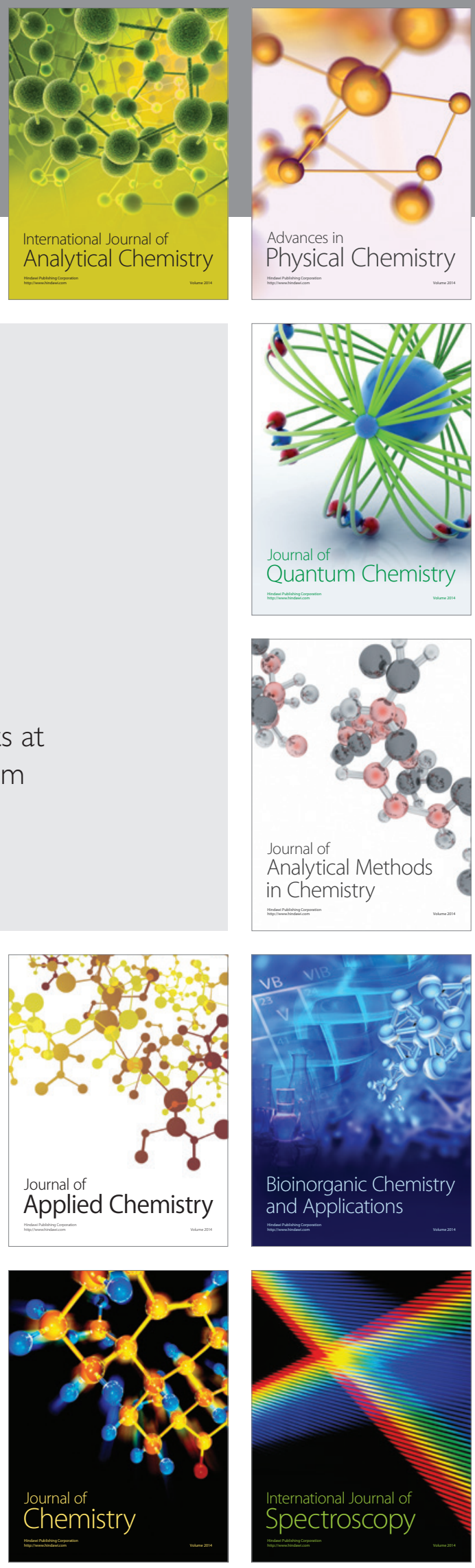